\title{
Pion-eta scalar-isovector 3-coupled channel amplitude fitted to branching ratios and threshold plus subthreshold parameters
}

\author{
Robert Kamiński ${ }^{1, a}$ and Łukasz Bibrzycki \\ 1 Institute of Nuclear Physics PAS, Kraków, Poland \\ ${ }^{2}$ Pedagogical University, Kraków, Poland
}

\begin{abstract}
The low energy (below $2 \mathrm{GeV}$ ) $\pi \eta$ channel interaction amplitude becomes an object of interest mainly because of the search for exotic mesons in just beginning to collect data detector GlueX in JLab. Finding and interpretation of expected weak signals from these states require a comparison with a very accurate amplitude containing standard $(q \bar{q})$ states i.e. $a_{0}(980)$ and $a_{0}(1450)$. The main problem in the determination of such amplitude is a total absence of data about the phases and inelasticities in the elastic and inelastic region. In addition, it is necessary to take into account the next two coupled higher channels $-K \bar{K}$ and $\pi \eta^{\prime}$. Presented here amplitude is based on separable potential model (working very well for the scalar-isoscalar $\pi \pi$ interactions) with only 9 free parameters. To determine such 3-coupled channel amplitude, the following information has been taken into account: experimental branching ratios and positions of both $a_{0}$ resonances, theoretical couplings, scattering length from ChPT and value of squared radius of the $\pi \eta$ form factor. Phase shifts, inelasticities and cross sections in all single and crossed channels are presented.
\end{abstract}

\section{Introduction}

Although the $\pi \eta$ scalar-isovector channel is very interesting there is no data on phase shifts and inelasticities. As constraints for constructed amplitudes can be used only positions of the $a_{0}(980)$ and $a_{0}$ (1450) relative ratios $\Gamma_{K K} / \Gamma_{\pi \eta}$ and $\Gamma_{\pi \eta^{\prime}} / \Gamma_{\pi \eta}$ from various analyses e.g. [1, 2], scattering length $a_{0}^{1} \approx 0.005-0.01 m_{\pi}^{-1}(\mathrm{ChPT})$ and radius of the $\pi \eta$ form factor $r^{2} \approx 0.1 \mathrm{fm}^{2}$ [3]. In some analyses also values of the couplings of the $a_{0}(980)$ and $a_{0}(1450)$ to three channels have been calculated $[1,4]$. They, however, differ significantly what reduces the possibility of their use in the analysis of amplitudes.

Recently group of theorists proposed simple model [1] in which the $a_{0}(1450)$ is a seed $q \bar{q}$ state which, due to coupling via meson loops (derivate and nonderivative terms added to Lagrangian), generates $a_{0}(980)$. Such dynamically generated $a_{0}(980)$ is therefore not $q \bar{q}$ state but rather mesonmeson molecule or $2 q 2 \bar{q}$ state. In another paper [3] unitary relations, chiral constraints and asymptotic QCD behavior have been used to calculate phase shifts for the $\pi \eta$ channel and radius of the $\pi \eta$ form factor. In series of papers (e.g. [4]) analyzed are high statistic Belle data on $\gamma \gamma \rightarrow \pi^{0} \eta$. As outcome the $a_{0}(980)$ appears as a $2 q 2 \bar{q}$ state, calculated are phase shifts and inelasticities in agreement with ChPT.

\footnotetext{
ae-mail: robert.kaminski@ifj.edu.pl
} 


\section{Method}

In presented here analysis separable potential approach is used. Several years ago it very well described the three coupled channel interactions $(\pi \pi, K \bar{K}$ and $\sigma \sigma)$ in the scalar-isoscalar channel $\left(0^{+} 0^{++}\right)$ [5]. Similar two channel analysis has been presented for the coupled $\pi \eta$ and $K \bar{K}$ channels in the scalarisovector channel [6]. Four parameters of the separable potential has been calculated analytically using four constraints being positions of poles of the $a_{0}(980)$ and $a_{0}(1450)$ resonances.

In that approach amplitudes $T$, potential $V$ and propagator $G$ are related by

$$
\begin{aligned}
<\mathbf{p}|T| \mathbf{q}>= & <\mathbf{p}|V| \mathbf{q}>+ \\
& +\int \frac{d^{3} s}{(2 \pi)^{3}}<\mathbf{p}|V| \mathbf{s}><\mathbf{s}|G| \mathbf{s}><\mathbf{s}|T| \mathbf{q}>
\end{aligned}
$$

where

$$
<\mathbf{p}\left|V_{i j}\right| \mathbf{q}>=\lambda_{i j} g_{i}(p) g_{j}(q), \quad g_{i}(p)=\sqrt{\frac{4 \pi}{m_{i}}} \frac{1}{p^{2}+\left(\beta_{i}\right)^{2}} .
$$

Reduced amplitude

$$
t=\lambda+\lambda I t \longrightarrow t=(1-\lambda I)^{-1} \lambda
$$

one can obtain calculating integrals

$$
I_{i i}=\int \frac{d^{3} s}{(2 \pi)^{3}} g_{i}(s) G_{i}(s) g_{i}(s)
$$

and Jost function is defined then as

$$
D(E)=\operatorname{det}(1-\lambda I(E)) \longrightarrow D\left(k_{1}, k_{2}\right)=D_{\pi \eta}\left(k_{1}\right) D_{K K}\left(k_{2}\right)-C\left(k_{1}, k_{2}\right) .
$$

The components are defined by

$$
D_{\pi \eta}\left(k_{1}\right)=1-\Lambda_{11} J_{11}\left(k_{1}\right), \quad D_{K K}\left(k_{2}\right)=1-\Lambda_{22} J_{22}\left(k_{2}\right) \quad C\left(k_{1}, k_{2}\right)=\Lambda_{12}^{2} J_{11}\left(k_{1}\right) J_{22}\left(k_{2}\right) .
$$

Then elements of the $S$ matrix are given by

$$
S_{11}=\frac{D\left(-k_{1}, k_{2}\right)}{D\left(k_{1}, k_{2}\right)}, \quad S_{22}=\frac{D\left(k_{1},-k_{2}\right)}{D\left(k_{1}, k_{2}\right)}, \quad S_{11} S_{22}-S_{12}^{2}=\frac{D\left(-k_{1},-k_{2}\right)}{D\left(k_{1}, k_{2}\right)} .
$$

Relation of these functions with amplitudes is:

$$
\begin{aligned}
& S_{11}=1-\frac{i k_{1} E_{1}\left(k_{1}\right)}{2 \pi} T_{11}\left(k_{1}, k_{2}\right) \\
& S_{22}=1-\frac{i k_{2} E_{2}\left(k_{2}\right)}{2 \pi} T_{22}\left(k_{1}, k_{2}\right) \\
& S_{12}=S_{21}=-\frac{i}{2 \pi} \sqrt{k_{1} E_{1}\left(k_{1}\right) k_{2} E_{2}\left(k_{2}\right)} T_{12}\left(k_{1}, k_{2}\right)
\end{aligned}
$$

therefore full $S$ matrix can be expressed by phase shifts $\delta$ and inelasticities $\eta$

$$
S=\left(\begin{array}{cc}
\eta e^{2 i \delta_{\pi \eta}} & i \sqrt{1-\eta^{2}} e^{i\left(\delta_{\pi \eta}+\delta_{K K}\right)} \\
i \sqrt{1-\eta^{2}} e^{i\left(\delta_{\pi \eta}+\delta_{K K}\right)} & \eta e^{2 i \delta_{K K}}
\end{array}\right) .
$$


The unitary amplitudes for interactions in two coupled channels $\pi \eta$ and $K K$ have been constructed in the wide energy range from the $\pi \eta$ threshold $(\approx 686 \mathrm{MeV})$ up to $\approx 1500 \mathrm{MeV}$. Worthy to note is that two poles corresponding to the $a_{0}(980)$, both very near the $K \bar{K}$ threshold have been found: at $991.5-i 33.6 \mathrm{MeV}$ on the $I I^{\text {nd }}$ Riemann sheet (i.e $I m k_{1}<0, \operatorname{Im}_{2}>0$ ) and $1005.0-i 24.5 \mathrm{MeV}$ on the $I I I^{r d}$ Riemann sheet (i.e $I m k_{1}<0, I m k_{2}<0$ ). Both play important role in construction of the amplitudes near the $1 \mathrm{GeV}$.

In order to expand analysis on the third $\pi \eta^{\prime}$ channel one has to introduce new constraints. It can be for example scattering length $a_{0}^{1} \approx 0.005-0.01 m_{\pi}^{-1}$, radius of the $\pi \eta$ form factor: $r^{2} \approx 0.1 \mathrm{fm}^{2}$ and information on couplings to the three channels: $\pi \eta, K \bar{K}$ and $\pi \eta^{\prime}$.

Three coupled channel amplitude has 9 parameters which determine full Jost function

$$
D\left(k_{1}, k_{2}, k_{3}\right)=D_{\pi \eta}\left(k_{1}\right) D_{K K}\left(k_{2}\right) D_{\pi \eta^{\prime}}\left(k_{3}\right)-C\left(k_{1}, k_{2}, k_{3}\right)
$$

where components for single channels have form defined in Eq. (6) and that coupling between all three channels is

$$
\begin{aligned}
C\left(k_{1}, k_{2}, k_{3}\right) & =\Lambda_{12}^{2} J_{11}\left(k_{1}\right) J_{22}\left(k_{2}\right)+\Lambda_{13}^{2} J_{11}\left(k_{1}\right) J_{33}\left(k_{3}\right)+\Lambda_{23}^{2} J_{22}\left(k_{2}\right) J_{33}\left(k_{3}\right)+ \\
& +\left(\Lambda_{12}^{2} \Lambda_{3}+\Lambda_{13}^{2} \Lambda_{2}+\Lambda_{23}^{2} \Lambda_{1}+\Lambda_{12} \Lambda_{13} \Lambda_{23}\right) J_{11}\left(k_{1}\right) J_{22}\left(k_{2}\right) J_{33}\left(k_{3}\right) .
\end{aligned}
$$

\section{Results}

Fitting all nine parameters to chosen constraints quite interesting amplitude has been obtained. Radius of the form factor has not been fitted but the value obtained integrating phase shifts in the elastic region

$$
<r^{2}>=\frac{6}{\pi} \int_{s_{0}}^{+\infty} d s \frac{\delta_{F^{\pi \eta}}(s)}{s^{2}}
$$

is $0.1 \mathrm{fm}^{2}$. Upper limit coming from integral to infinity gives $0.15 \mathrm{fm}^{2}$ very well agrees with other predictions e.g. [3] $0.12-0.18 \mathrm{fm}^{2}$.

Figures 1-9 present phase shifts, inelasticities and cross sections for found amplitude. If possible they are compared with the corresponding ones for the 2-channel amplitude [6].

On figure 1 is seen that in the three channel model the $\pi \eta$ phase shifts show characteristic 180 degree jumps in vicinity of $1 \mathrm{GeV}$ and $1.5 \mathrm{GeV}$. Of course they correspond to both $a_{0}$ mesons. In contradiction to two channel model the latter jump is more visible. It is due to possible new coupling - to the $\pi \eta^{\prime}$ channel.

Inelasticities in the three channel case presented on figure 2 show characteristic small minimum above $1.6 \mathrm{GeV}$ caused by presence of the third channel. Much smaller, than in the two channel model, minimum just above $1 \mathrm{GeV}$ shows that coupling of the $a_{0}(980)$ to $\pi \eta$ channel is smaller because now, this meson can decay also to the third channel $\pi \eta^{\prime}$.

Completely different $K \bar{K}$ phase shifts on figure 3 show that interference between phases of components in Jost functions (5) and (10) is very different.

Inelasticities in the $K \bar{K}$ channel on figure 4 are similar to those on figure 2 (they are identical below the $\pi \eta^{\prime}$ threshold) except the luck of small minimum near the $1.6 \mathrm{GeV}$. This can be caused just by smaller coupling of the $a_{0}(980)$ to the $K \bar{K}$ channel.

Presented on figure 5 elastic cross sections $\pi \eta \rightarrow \pi \eta$ have narrower maximum in the three channel model. It is natural consequence of presence of the $\pi \eta^{\prime}$ channel.

Similarly to inelasticities on figure 4, the elastic cross section $K \bar{K} \rightarrow K \bar{K}$ on figure 6 does not reveal significant structure near $1 \mathrm{GeV}$. Narrower and smaller maximum near the $1.5 \mathrm{GeV}$ in the 3 

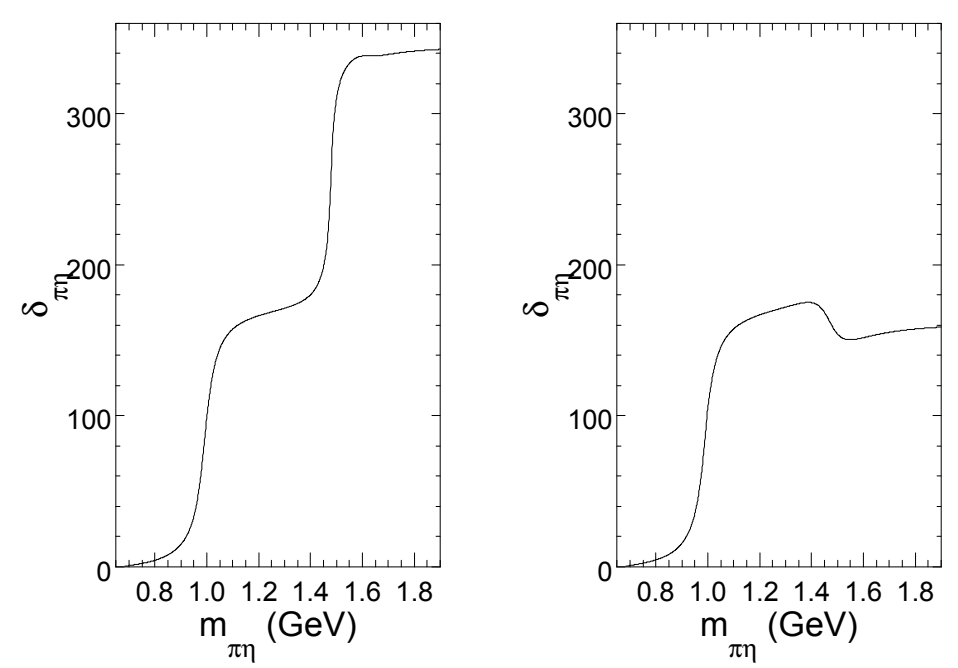

Figure 1. Phase shifts in the $\pi \eta$ channel for 3-channel model (left) and 2-channel one (right).
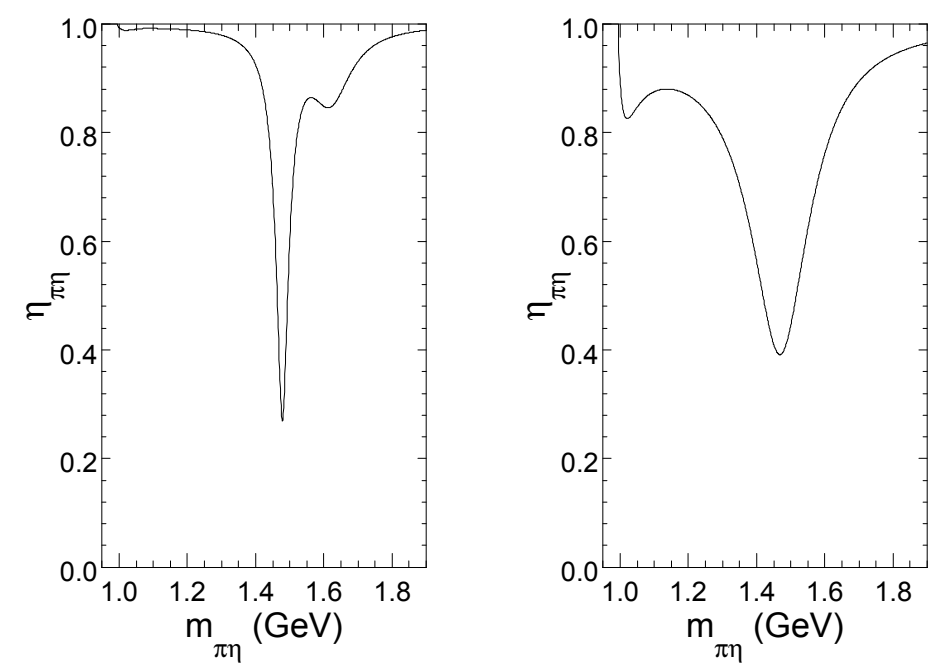

Figure 2. Inelasticities in the $\pi \eta$ channel for 3-channel model (left) and 2-channel one (right).

channel case shows that coupling of the $a_{0}(1450)$ to the $K \bar{K}$ is much smaller. Again it is natural consequence of its coupling to the third channel.

Similar structure for the transition cross section $\pi \eta \rightarrow K \bar{K}$ is seen on figure 7. New maximum near the $1.6 \mathrm{GeV}$ corresponds to small minimum of the $\pi \eta$ inelasticity on figure 2 .

Characteristic maximum of the elastic $\pi \eta^{\prime}$ cross section near $1.6 \mathrm{GeV}$ on figure 8 is due to coupling of the $a_{0}(1450)$ to this channel. Much smaller corresponding maximum in the transition cross section 

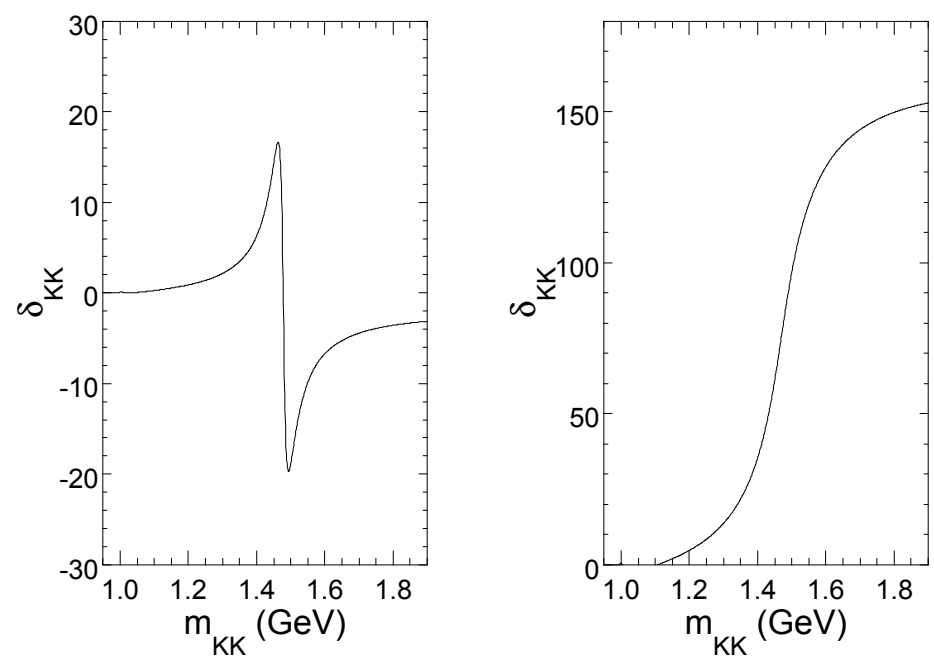

Figure 3. Phase shifts in the $K \bar{K}$ channel for 3-channel model (left) and 2-channel one (right).
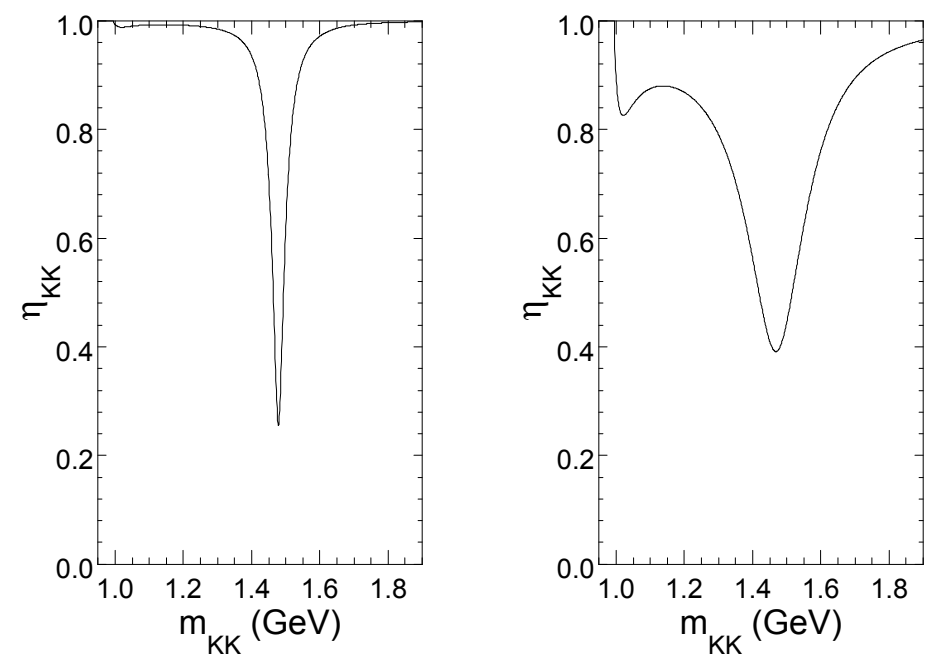

Figure 4. Inelasticities in the $K \bar{K}$ channel for 3-channel model (left) and 2-channel one (right).

$\pi \eta \rightarrow \pi \eta^{\prime}$ shows that this meson decays mostly to $\pi \eta^{\prime}$ channel. Small maximum of this transition near $1.5 \mathrm{GeV}$ and its non zero value below this energy is consequence of coupling of the $a_{0}(980)$ to both the $\pi \eta$ and $\pi \eta^{\prime}$ channels.

The $\pi \eta^{\prime}$ phase shifts on figure 9 present typical resonance structure near the $1.6 \mathrm{GeV}$ and inelasticities in the same region - small minimum being signal of coupling the $a_{0}(1450)$ to this channel. 

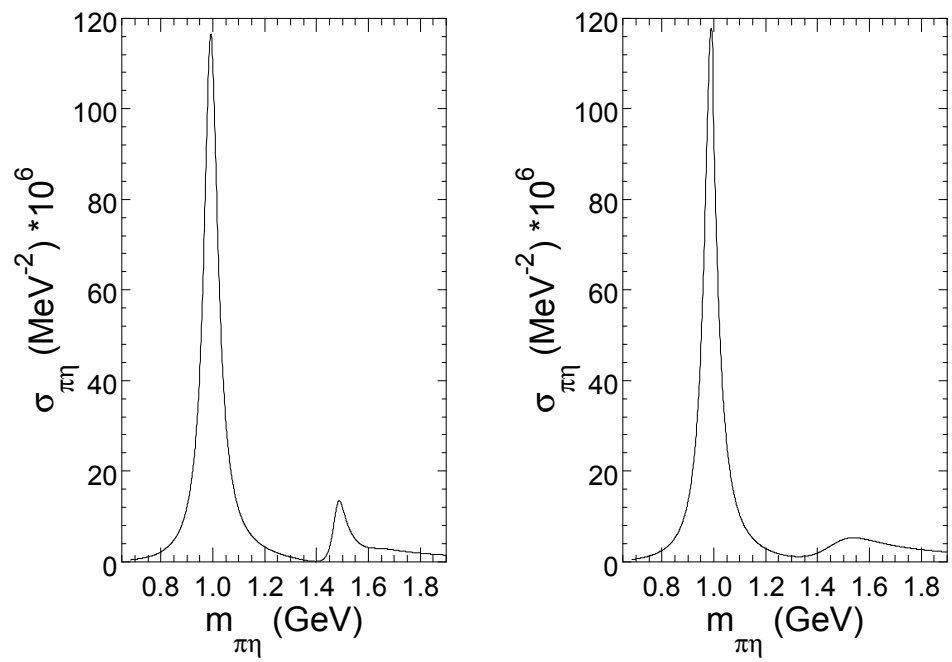

Figure 5. Cross sections for the $\pi \eta$ channel in the 3-channel model (left) and 2-channel one (right).
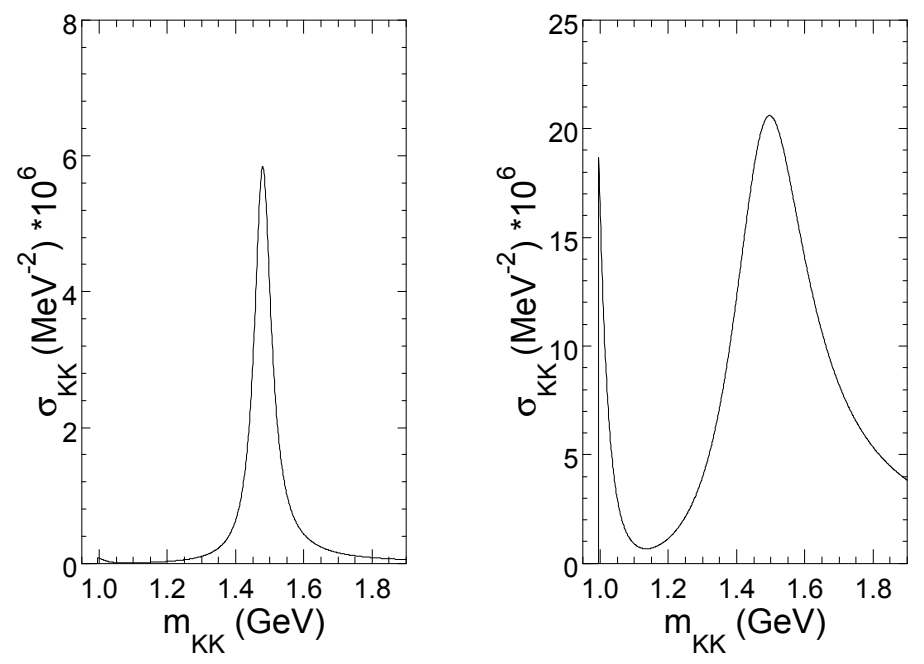

Figure 6. Cross sections for the $K \bar{K}$ channel in the 3-channel model (left) and 2-channel one (right).

\section{Conclusions}

Although, from experimental point of view, the scalar-isovector $\pi \eta$ channel is the one of the least known channel of interactions of light mesons one can construct three-coupled channel amplitude using constraints like positions of poles corresponding to the $a_{0}(980)$ and $a_{0}(1450)$ states, scattering length and relative couplings for the $\pi \eta, K \bar{K}$ and $\pi \eta^{\prime}$ channels. Three coupled channel amplitude fitted 

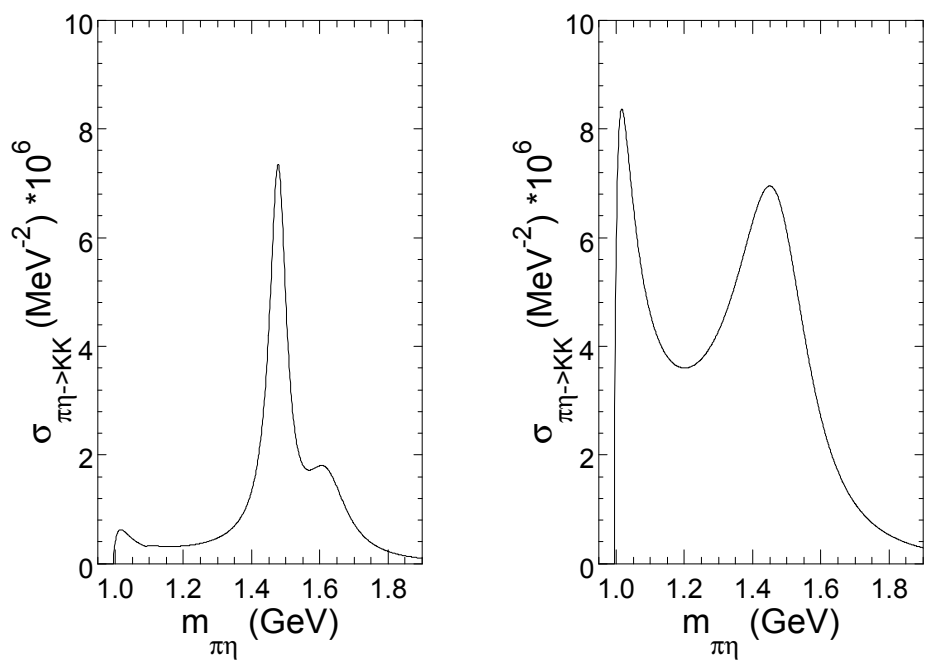

Figure 7. Transition cross sections $\pi \eta \rightarrow K \bar{K}$ in the 3-channel model (left) and 2-channel one (right).
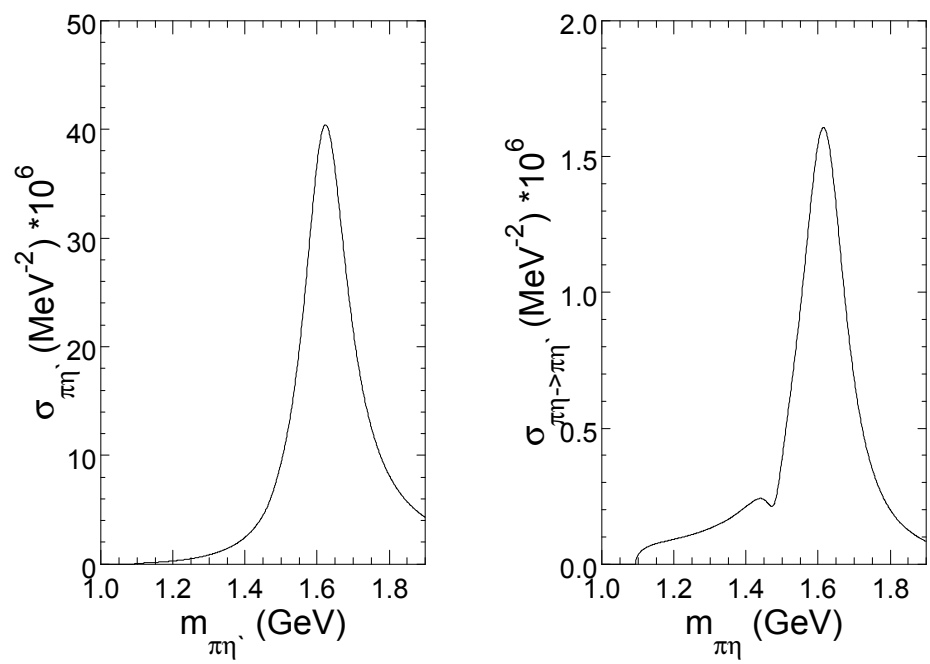

Figure 8. Cross sections for the $\pi \eta^{\prime}$ channel (left) and transition $\pi \eta \rightarrow \pi \eta^{\prime}$ (right) in the 3-channel model.

to these constraints shows importance of the third - $\pi \eta^{\prime}$ channel. In calculation of the any physical quantity, for example radius of the form factor or couplings of the $a_{0}$ resonances, one should take into account three coupled channels. This can be very useful in construction of final state interaction amplitudes needed in e.g. searches of the exotic mesons at JLab. Needed are, however, new analyses which could deliver phase shifts and inelasticities at least in the first two channels: $\pi \eta$ and $K \bar{K}$. Having such amplitudes very helpful can be Roy-like dispersion relations with imposed crossing symmetry. 

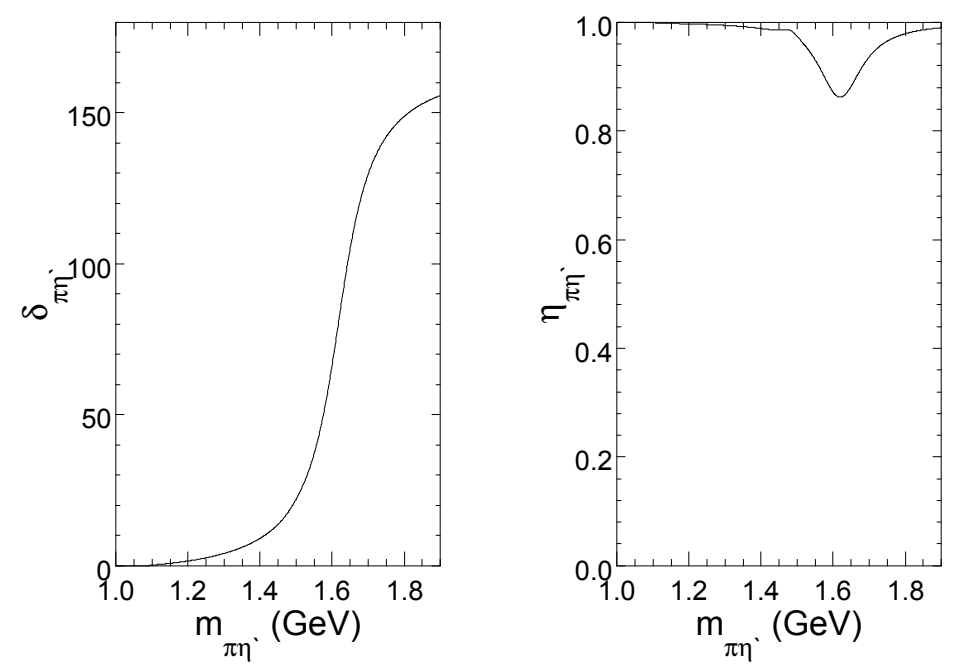

Figure 9. Phase shifts for (left) and inelasticities for the $\pi \eta^{\prime}$ channel in the 3-channel model.

\section{Acknowledgments}

This work has been partially supported by the Polish Science Center (NCN) Grants No. Dec2013/09/B/ST2/04382

\section{References}

[1] T. Wolkanowski, F. Giacosa and D. H. Rischke, Phys. Rev. D93, 014002 (2016).

[2] C. Patrignani et al. (Particle Data Group), Chin. Phys. C, 40, 100001 (2016).

[3] M. Albaladejo and B. Moussallam, Eur. Phys. J. C75, 488 (2015).

[4] N. N. Achasov and G. N. Shestakov, Phys. Rev. D81, 094029 (2010).

[5] R. Kamiński, L. Leśniak and B. Loiseau, Eur. Phys. J. C9, 141 (1999).

[6] A. Furman, L. Leśniak, Phys.Lett. B538, 266 (2002). 